\title{
ADSORPTION: AN ECONOMICAL METHOD FOR THE REMOVAL OF HEAVY METALS FROM WATER
}

\author{
Dr. Sureshkumar Halnor \\ Department of Chemistry Doctor Vithalrao Vikhe Patil College of Engineering, \\ Ahmednagar (M.S.), India.
}

\begin{abstract}
This study reviews various techniques available for removal of heavy metals from waste water including adsorption technique as economical technique. Different adsorbents used for removal different heavy metals are discussed

Key words: low cost materials, industrial waste water, adsorbents , adsorption and heavy metals
\end{abstract}

\section{INTRODUCTION}

Water is very essential for human being, microorganism and living things, plants and various industrial processes etc. Out of the many sources of water the purest source of water is the rain water. But while its journey towards earth it gets mixed with various substances and materials and due to which it gets converted into impure water. As many properties of this water get altered due to impurities so it becomes inconvenient to our daily use including drinking, washing, industrial processes etc.

International World Water Dayis celebrated on $22^{\text {nd }}$ March of every year to realize the importance of water to the public. On this day during its celebration we realize the importance of water, water management by all components like individuals, governments, various organizations, communities etc., water scarcity, why and how to save and recycle the water.

Today's problems about water are mainly water pollution, scarcity of water, shortage of proper drinking water and decrease in level of water tables.
As there is direct effect of quality of water on human health so it is very important to maintain the quality of water. Water quality is checked and confirmed by different tests of water.

There are many substances and materials are found in impure water. Among various substances and materials found in water it is essential to study heavy metals mainly because many of them are toxic and affect the human life and health. And after exceeding their tolerance limit the heavy metals should be removed from the water.

Many processes have been used for the removal of heavy metals from waste waters, such as chemical precipitation, coagulation, solvent extraction, membrane separation, ion exchange and adsorption ${ }^{1}$.For dilute concentrations of the metals ion exchange, reverse osmosis and adsorption techniques can be used. But among these ion exchange, reverse osmosis is costly. So as alternative to various methods for removal of heavy metals is adsorption method. This method has been tested by many researchers by using different adsorbents. 
Heavy metals: Generally, found heavy metals in industrial wastewater are $\mathrm{Pb}, \mathrm{Cr}, \mathrm{Hg}, \mathrm{As}, \mathrm{Ni}$, $\mathrm{Cu}$ etc $^{2}$.Their details are as below,

\begin{tabular}{|c|c|c|c|}
\hline $\begin{array}{l}\text { Heavy } \\
\text { metal }^{1}\end{array}$ & $\begin{array}{l}\text { MPL (mg L- } \\
{ }_{1}^{-1}\end{array}$ & Sources $^{3}$ & Adverse effects ${ }^{3}$ \\
\hline $\mathrm{Pb}$ & 0.05 & $\begin{array}{l}\text { Glass, lead paint, printing, } \\
\text { Batteries } \\
\text { manufacturing industries }\end{array}$ & $\begin{array}{l}\text { Destruction in kidney, } \\
\text { reproductive system, liver, } \\
\text { brain and central nervous } \\
\text { system, anemia,headache. }{ }^{4}\end{array}$ \\
\hline $\mathrm{Cr}$ & 0.05 & $\begin{array}{l}\text { Electroplating, tanning, } \\
\text { dyeing, } \\
\text { leather, textile industries. }\end{array}$ & $\begin{array}{l}\text { Carcinogenic, liver damage, } \\
\text { skin } \\
\text { irritation, ulcer,edema. }^{5}\end{array}$ \\
\hline $\mathrm{Hg}$ & 0.1 & $\begin{array}{l}\text { Paints, pulp and paper, oil } \\
\text { refining, } \\
\text { rubber processing and } \\
\text { fertilizer industries Batteries, } \\
\text { thermometers, fluorescent } \\
\text { light tubes and high } \\
\text { intensity street lamps, } \\
\text { pesticides, cosmetics, } \\
\text { pharmaceuticals industries }\end{array}$ & $\begin{array}{l}\text { Mercury is generally } \\
\text { considered to be one of the } \\
\text { most toxic metal found in the } \\
\text { environment. It } \\
\text { causes mental retardation, } \\
\text { genetic defects, teratogenic } \\
\text { effects. Mercury causes } \\
\text { impairment of pulmonary } \\
\text { function and kidney, chest } \\
\text { pain }{ }^{6}\end{array}$ \\
\hline As & 0.06 & $\begin{array}{l}\text { Ceramic, fertilizers, } \\
\text { detergents, } \\
\text { metallurgical, dyes, glass } \\
\text { wares, } \\
\text { pesticides industries }\end{array}$ & $\begin{array}{l}\text { Vomiting, diarrhea, black } \\
\text { foot disease, Cancer.. }\end{array}$ \\
\hline $\mathrm{Ni}$ & 0.1 & $\begin{array}{l}\text { Silver refineries, } \\
\text { electroplating } \\
\text { industry, metal finishing and } \\
\text { forging, batteries } \\
\text { manufacturing, mining } \\
\text { industries. }\end{array}$ & $\begin{array}{l}\text { Dermatitis, cancer of lungs, } \\
\text { Acute poisoning of } \mathrm{Ni} \\
\text { (II) causes headache, } \\
\text { dizziness, nausea vomiting, } \\
\text { chest pain, extreme } \\
\text { weakness. }\end{array}$ \\
\hline $\mathrm{Cu}$ & 2.0 & $\begin{array}{l}\text { As contaminant in food } \\
\text { especially } \\
\text { shellfish, liver, mushroom, } \\
\text { nuts, } \\
\text { chocolate, PCB designing, } \\
\text { electroplating industry. }\end{array}$ & $\begin{array}{l}\text { Carcinogenic, } \\
\text { Hemochromatosis, } \\
\text { gastrointestinal problems, } \\
\text { accumulation in the kidneys, } \\
\text { brain, skin and heart. }^{8}\end{array}$ \\
\hline
\end{tabular}

[MPL: maximum permissible limit] 
Techniques: Different techniques which have been studied for the removal of heavy metals from the industrial wastewater are as below,

\begin{tabular}{|c|c|c|}
\hline Techniques $^{9}$ & Advantages $^{9}$ & Disadvantages $^{9}$ \\
\hline Electrodialysis & Pure effluent & $\begin{array}{l}\text { Expensive, Formation of } \\
\text { metal hydroxides which clog } \\
\text { the membrane. }\end{array}$ \\
\hline $\begin{array}{l}\text { Chemical } \\
\text { precipitation }\end{array}$ & Cheap, Simple & $\begin{array}{l}\text { Generates sludge, Difficult } \\
\text { separations }\end{array}$ \\
\hline Evaporation & Pure effluent & $\begin{array}{ll}\text { Generates } & \text { sludge's, } \\
\text { Expensive } & \end{array}$ \\
\hline Reverse Osmosis & Pure effluent & Expensive, High pressures \\
\hline $\begin{array}{l}\text { Chemical } \\
\text { oxidation/reduction }\end{array}$ & Mineralization & Chemicals required \\
\hline Electrochemical & Metal recovery & $\begin{array}{l}\text { Expensive, For high } \\
\text { concentrations }\end{array}$ \\
\hline Ion exchange & Metal recovery & $\begin{array}{l}\text { Sensitive to particles, } \\
\text { Expensive }\end{array}$ \\
\hline
\end{tabular}

From all above advantages and disadvantages of various techniques adsorption technique seem to be suitable for the heavy metals removal from the industrial wastewater.

Adsorption: This technique is found superior as compared to all other techniques as it has following advantages ${ }^{9}$,

- Easy to operate

- Cheap adsorbent

- Insensitivity to toxic pollutants

- No chemical or biological sludge

- Economical

- Very simple technique

- Less space required

- Low cost

- Natural/eco-friendly adsorbents

- High efficiency
- Possibility of metal recovery

- Produce high quality treated water

- Simple equipment

Adsorbents: Various adsorbents have been used for removal of heavy metals from polluted and industrial wastewater by various researchers. Adsorbents which should be used for this purpose should be low cost, abundantly available, environmental friendly and easily available. Literature survey shows that such adsorbents are various plant materials, waste building materials, various biological materials, byproducts and waste materials from different industries etc. Their few examples are Algae, Jute, Cashew nut shells, Date tree leaves, Ashoka leaf, Jute stick, Green tea, Jambhool, Ratrani leaf, Coconut husk, Tea waste, Almond husk, Cassia Siamea, Coffee, 
Potato husk, Sunnhemp, Apple Pomace, Syzygium cumini, Prosopis spicegera, activated red mud, Hydroxides of aluminium and iron, ores, hairs, albumin, activated carbon, feathers, bagasse, wool, waste rubber etc. These adsorbents can remove $\mathrm{Fe}, \mathrm{Cd}, \mathrm{Hg}, \mathrm{Cr}, \mathrm{Ni}, \mathrm{Cu}, \mathrm{As}, \mathrm{Co}$ etc. like heavy metals from polluted and industrial waste water.

Few pre-treated adsorbents also used by some researchers for the removal of heavy metals from polluted and industrial waste water and their use showed increase in adsorption efficiency but in some cases cost of whole process increases. Various chemicals those are used for this purpose are phosphoric acid, Potassium hydroxide, Sulphuric acid, calcium hydroxide, nitric acid, sodium hydroxide, sodium carbonate, hydrochloric acid etc.

Following few examples shows how the pre-treated adsorbents increases adsorption efficiency.

\begin{tabular}{|c|c|}
\hline \multicolumn{2}{|c|}{$\mathrm{Cr}^{10} \mathrm{Removal}^{2}$} \\
\hline Jambhool leaf & Jambhool leaf powder \\
powder(Untreated) & $\left(\mathrm{HNO}_{3}\right.$ treated $)$ \\
$69.2 \%$ & $98.4 \%$ \\
\hline
\end{tabular}

\begin{tabular}{|c|c|}
\hline \multicolumn{2}{|c|}{$\mathrm{Cu}^{11}$ Removal } \\
\hline $\begin{array}{c}\text { Nerium Indicum } \\
\text { (Untreated) }\end{array}$ & $\begin{array}{l}\text { Nerium Indicum }(\mathrm{HCl} \\
\text { \& NaOH treated }) \\
71 \%\end{array}$ \\
$80 \%$ \\
\hline
\end{tabular}

\begin{tabular}{|c|c|}
\hline \multicolumn{2}{|c|}{$\mathrm{Fe}^{12}$ Removal } \\
\hline Syzygium & Syzygium cumini \\
cumini(Untreated) & $\left(\mathrm{HNO}_{3}\right.$ treated $)$ \\
$73.62 \%$ & $94.21 \%$ \\
\hline
\end{tabular}

\section{Applications of adsorption:}

This adsorption method can be used for recovery of metals from polluted and industrial waste water, recovery of dyes from solution, chromatographic analysis, purification of air, clarification of sugar liquid, heavy metals removal from polluted and industrial waste water, impurities removal from crude oil, purification, separation of gas mixture, softening of hard water, decolourisation etc.

\section{CONCLUSION}

From all above adsorption seems to be the best substitute for the other conventional techniques to remove the heavy metals from the polluted and wastewater.

\section{REFERENCES}

(1) Sureshkumar Halnor et al, Journal of Applicable Chemistry,2013, 2 (3): 475-485.

(2)Dr. Sureshkumar Halnor, IJGHC, Dec 2018 Feb.2019; Sec. A; Vol.8, No.1, 118-122.

(3) Sureshkumar Halnor,International Journal of Application or Innovation in Engineering \&

Management,Oct. 2015, Vol 4, Issue 10, 19-22.

(4) N. Kannan, P. Sarojini, Ind. J. Env. Prot., 2010,30(5), 404-408.

(5) P.C. Mane, A.B. Bhosale, C.M. Jangam, S.V. Mukate, J. Nat.Prod. Plant Resour., 2011,1(1), 75-80.

(6) C. Namasivayam, K. Periasamy, Water Res., 1993, 27, 1663-1668.

(7) A.R. Quaff, M.M. Ashhar, J. of Indian Water Works Association, 2005, Vol. 37 No. I, 63-69.

(8) A.K. Maurya, R.K. Sharma, A. Kumar, P.E. Joseph, Ind.J. Env. Prot., 2007,27(3), 272-275

(9) Sureshkumar Halnor, International Journal of Scientific Research and Reviews, April-

June. 2019,8(2), 2809-2815.

(10) Sureshkumar Halnor, M. Farooqui, A. Taher, Milind Ubale, Int. J. of Green \& Herbal

Chem.,2012, Vol.1, No.2, 169-175.

(11) A. Taher, Mazahar farooqui, Maqdoom Farooqui, J. of Chemical, Biological \& Physical Sciences, 2012,Vol.2, No.2, 661-667.

(12) Sureshkumar Halnor, R. Dandge, Milind Ubale, Pelagia Research Library, 2012, 3(6), 1444-1449. 